\title{
Magneto-Rheological Dynamometer Dynamic Model Research
}

\author{
Tian Pengfei", Luo Yiping \\ School of Automotive Engineering, Shanghai University of Engineering Science, Shanghai, P.R. China
}

Email address:

tianpf.2009@163.com (Tian Pengfei)

To cite this article:

Tian Pengfei, Luo Yiping. Magneto-Rheological Dynamometer Dynamic Model Research. International Journal of Materials Science and Applications. Vol. 4, No. 4, 2015, pp. 241-245. doi: 10.11648/j.ijmsa.20150404.13

\begin{abstract}
This article simply introduces a new material magneto-rheological fluid (MRF) and its widely used, and developed a new type of magneto-rheological dynamometer based on the rheological properties of MRF. This dynamometer introduces magneto-rheological fluid as the working medium, and using the shear yield stress of magneto-rheological fluid in a magnetic field to transmit torque. The paper explored the working mechanism of MR dynamometer, established the torque transmission model of MR dynamometer, and analyzed the flow properties of MRF in the dynamometer under steady-state based on Bingham model and fluid momentum equation.
\end{abstract}

Keywords: MRF, Dynamometer, Bingham Model, N-S Equation

\section{Introduction}

MRF (magneto-rheological fluid) is a kind of new smart material which rheological properties can change drastically when affected by external magnetic fields. It is the suspension of micron-sized magnetic particles dispersed in a non-magnetic liquid (mineral oil, silicone oil, etc.). It performances as good flowing liquid state in the absence of external magnetic field, after applying a external magnetic field, its viscosity can be increased by more than two orders of magnitude in milliseconds, and presents a similar mechanics properties of solids, and the viscosity change is continuous and reversible. Because of MRF reversible, rapid response and easy to control, it is widely used in the automotive, construction, medical, fitness equipment, etc. $[1,2]$.

Magneto-rheological fluid is mainly composed of the following three parts [3]:

1) Magnetic particles as a dispersed phase;

2) Foundation liquid as the carrier of the dispersed phase, also known as the carrier liquid;

3) Polymer additives, In order to improve the performance of MRF, including Surfactant (used to enhance magneto-rheological effect), dispersing agent (to prevent particle agglomeration), Stabilizers and lubricants (to prevent sedimentation), etc.

MRF dynamometer is the device that using the shear stress generated by MR effect of magneto-rheological fluid to transmit torque. At present, domestic and international research on magneto-rheological dynamometer is not a lot, most of the research about the application of magneto-rheological fluid are concentrated in the MR damper, MR clutch, MR shock absorber, MR brake, etc. Zheng Jun analyzed the steady flow of magneto-rheological fluids in the transmission apparatus [4]. Kamath analyzed the pressure-driven flow of magneto-rheological fluids in the MR damper [5]. Li Hongnan, LI Zhanwei summarized the status of researching on dynamic models of MR Damper, and compared the advantages and disadvantages of various dynamic models [6, 7]. Liu Zhongliang established a dynamic model of a certain type of MR damper [8]. Meng Weijia designed a kind of double flats MR clutch and established the dynamic model of the MR clutch [9]. Hu Hongsheng established the dynamic model of the MR shock absorber under impact load [10]. Liu Cheng, He Jianmin analyzed the viscoplastic flow of magneto-rheological fluids in the cylindrical MR brake [11, 12]. Tian Chaoyang established the dynamic model of disc MR brake [13]. The paper explored the working mechanism of MR dynamometer, established the torque transmission model of MR dynamometer, and analyzed the steady flow of magneto-rheological fluids in the MR dynamometer under steady-state based on Bingham model and fluid momentum equation. 


\section{MRF Dynamometer Structure Principle}

\subsection{Rheological Properties of MRF}

Magneto-rheological fluid present a Newtonian fluid properties of low viscosity in zero magnetic field, after applying an external magnetic field, it will be in a very short period of time (a few milliseconds) showing a high viscosity, low liquidity Bingham fluid properties [14]. Its constitutive equation is:

$$
\begin{gathered}
\begin{cases}\tau=\tau_{\mathrm{B}} \operatorname{sgn}(\gamma)+\eta \gamma & |\tau| \geq \tau_{B} \\
\gamma=0 & |\tau|<\tau_{B}\end{cases} \\
\gamma=r \frac{d \omega}{d r}
\end{gathered}
$$

Where, $\tau$ is the shear stress of MRF, $\tau_{\mathrm{B}}$ is the shear yield stress of MRF, $\eta$ is the dynamic viscosity of MRF, $\gamma$ is the shear strain rate of MRF.

From the MRF constitutive equation, when $\tau \geq \tau_{\mathrm{B}}$, MRF cannot yield; when $\tau<\tau_{B}$, MRF yield, the interface between yield zone and no yield zone is the critical yield surface.

In addition, the study found that, when the magnetic particles in magneto-rheological fluids are not completely saturated, the shear stress $\tau$ can be written as the power function of magnetic induction $B[15,16]$.

$$
\tau_{B}=\alpha B^{n}
$$

Where: $\alpha$ is a constant; Exponent $n$ is usually between 1 and 2, specific values depends on the material of the liquid.

\subsection{MRF Dynamometer Structure}

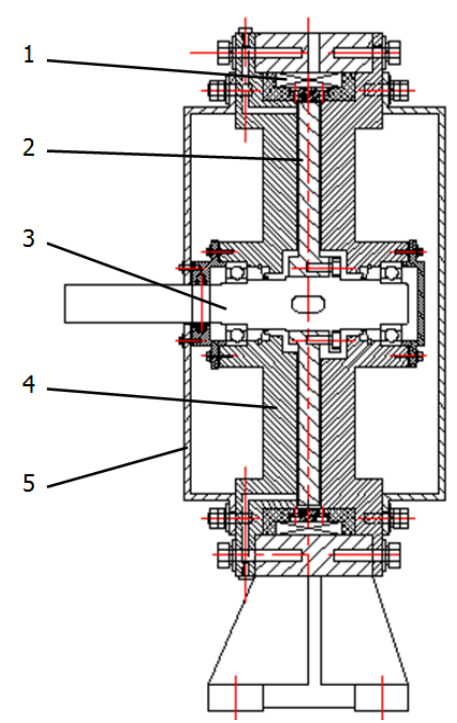

Fig. (1). MRF dynamometer structure.

\footnotetext{
1. Coils, 2. Active turntable

3. Drive shaft, 4. Casing

5. Water jacket
}

It is the structure diagram of MRF dynamometer body shown in Figure (1). Drive shaft connected with the engine, the active disc fixed to the drive shaft, Drive shaft made of non-magnetic material, the active turntable and the casing made of magnetically conductive material. Coils are radially surrounded outside the casing. The gap between the casing and the turntable is the magneto-rheological fluids workspace. When the coil is energized, the magnetic field generated axially through the casing, active disc and Magneto-rheological fluids, then magneto-rheological fluids generate MR effect, the turntable will shear magneto-rheological fluids when it rotates. Two water jackets installed in the shell on both sides, the jackets are connected with water pump and radiator to cool the MRF dynamometer.

\subsection{MRF Dynamometer Working Principle}

MRF dynamometer is a new dynamometer developed by magneto-rheological fluid responsive, reversible and easy to control features. Magneto-rheological dynamometer mainly uses the MRF shear work mode, and its structure using the disc type. When the coil is not energized, the magneto-rheological fluid exhibits Newtonian fluid state, the active disk rotation is only affected by the viscous resistance of the fluid; When the coil is energized, the work area generates a magnetic field in a direction along the axis of rotation, the magneto-rheological fluid exhibits Bingham fluid state. The magnetic particles in magneto-rheological fluids form a magnetic chain end to end in the direction of magnetic field, the magnetic chain will be cut when the turntable rotates. The torque that dynamometer can absorb is determined by the shear strength of magnetic chain, the shear strength increase with the magnetic field strength increases before the magnetic chain reaching saturation, when the excitation current is large enough, the magnetic chain saturated, the shear strength is not increase with the magnetic field strength increases.

\section{MRF Dynamometer Torque Transmission}

\subsection{Torque Model}

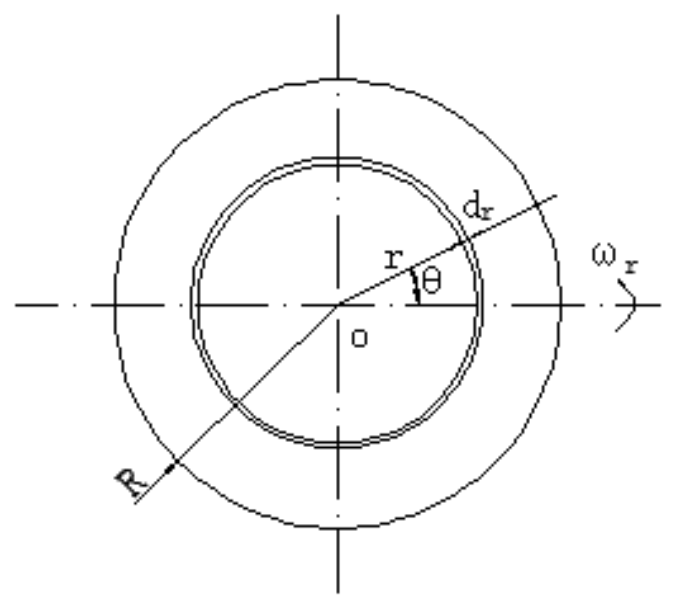

Fig. (2). MRF dynamometer Torque model. 
As shown in Figure (2), take an infinitesimal any angle on the disc where radius is $r$.

\subsection{Torque Calculation}

Shear stress at the infinitesimal is:

$$
d F=\tau \cdot d A=\tau 2 \pi r d r
$$

Torque at the infinitesimal is:

$$
d T=r \cdot d F=2 \pi r^{2} \tau d r
$$

Shear strain rate is:

$$
\gamma=r \cdot \frac{\omega_{2}-\omega_{1}}{h}
$$

Where, $h$ is the width of work space between active turntable and casing, $r$ is the radius at the infinitesimal, $\omega_{1}$, $\omega_{2}$ are respectively the angular velocity of the casing and the turntable.

Substituted formula (1), (6) into equation (5), and then integrating formula (5):

$$
\begin{aligned}
T & =\int_{r_{1}}^{r_{2}} 2 \pi r^{2} \tau d r=2 \pi \int_{r_{1}}^{r_{2}} r^{2}\left(\tau_{B}+\eta r \frac{\omega_{2}-\omega_{1}}{h}\right) d r \\
& =\left.\left(\frac{2 \pi}{3} \tau_{B} r^{3}+\frac{1}{2 h} \pi \eta \omega r^{4}\right)\right|_{r_{1}} ^{r_{2}} \\
& =\frac{2 \pi}{3} \tau_{B}\left(r_{2}^{3}-r_{1}^{3}\right)+\frac{\pi \eta \omega}{2 h}\left(r_{2}^{4}-r_{1}^{4}\right)
\end{aligned}
$$

As can be seen from the above equation, the torque that MRF dynamometer absorbed consists of two parts: one is $T_{B}$ that generated by magneto-rheological fluids shear yield stress; the second is the viscous torque $T_{\eta}$ that generated by the own viscosity of magneto-rheological fluids.

\subsection{Model Assumptions}

The following assumptions used in establishing the magneto-rheological flow model:

1) Magneto-rheological fluid is an incompressible fluid;

2) Magneto-rheological fluid flow in steady state;

3) Magneto-rheological fluid does not flow in the axial and radial;

4) The magnetic field strength is evenly distributed in the work space.

\subsection{Model Analysis}

As shown in Figure (3), establishing a cylindrical coordinate system, Z-axis is the straight line where the axis of rotation located in, the turntable radius is $r$, and the turntable angular velocity is $\omega$.

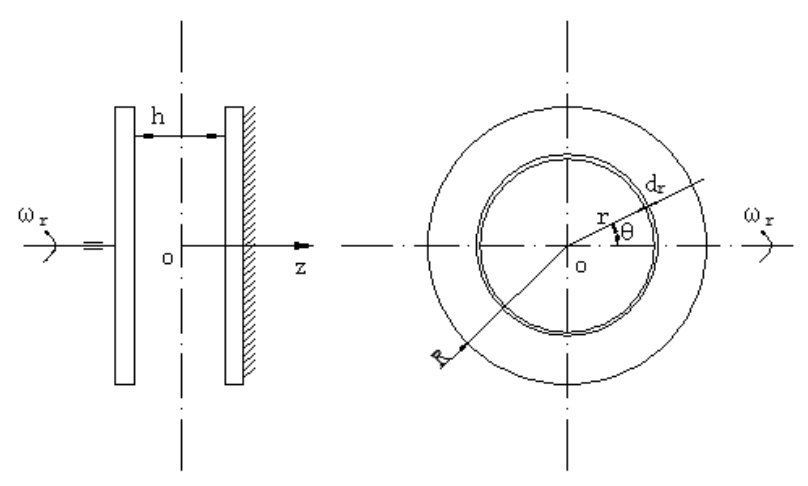

Fig. (3). cylindrical coordinate system.

Fluid continuity equation is:

$$
\frac{\partial u_{r}}{\partial r}+\frac{u_{r}}{r}+\frac{1}{r} \frac{\partial u_{\theta}}{\partial \theta}+\frac{\partial u_{z}}{\partial z}=0
$$

Fluid N-S equation in direction $r$ and $\theta$ is:

\section{Magneto-Rheological Fluid Flow Model}

$$
\begin{gathered}
\frac{\partial u_{r}}{\partial t}+u_{r} \frac{\partial u_{r}}{\partial r}+\frac{u_{\theta}}{r} \frac{\partial u_{r}}{\partial z}-\frac{u_{\theta}^{2}}{r}=f_{r}-\frac{1}{\rho} \frac{\partial p}{\partial r}+v\left(\frac{\partial^{2} u_{r}}{\partial r^{2}}+\frac{1}{r} \frac{\partial u_{r}}{\partial r}+\frac{1}{r^{2}} \frac{\partial^{2} u_{r}}{\partial \theta^{2}}+\frac{\partial^{2} u_{r}}{\partial z^{2}}-\frac{2}{r^{2}} \frac{\partial u_{\theta}}{\partial \theta}-\frac{u_{r}}{r^{2}}\right) \\
\frac{\partial u_{\theta}}{\partial t}+u_{r} \frac{\partial u_{\theta}}{\partial r}+\frac{u_{\theta}}{r} \frac{\partial u_{\theta}}{\partial \theta}+u_{z} \frac{\partial u_{\theta}}{\partial z}+\frac{u_{r} u_{\theta}}{r}=f_{\theta}-\frac{1}{\rho r} \frac{\partial p}{\partial \theta}+v\left(\frac{\partial^{2} u_{\theta}}{\partial r^{2}}+\frac{1}{r} \frac{\partial u_{\theta}}{\partial r}+\frac{1}{r^{2}} \frac{\partial^{2} u_{\theta}}{\partial \theta^{2}}+\frac{\partial^{2} u_{\theta}}{\partial z^{2}}-\frac{u_{\theta}}{r^{2}}+\frac{2}{r^{2}} \frac{\partial u_{r}}{\partial \theta}\right)
\end{gathered}
$$

Each velocity component of MRF is:

$$
\begin{aligned}
& u_{\theta}=r \cdot \omega(r) \\
& u_{r}=u_{z}=0
\end{aligned}
$$

As is steady flow:

$$
\frac{\partial u}{\partial t}=0
$$

Body force:

$$
\begin{aligned}
& f_{r}=-g \cos \theta \\
& f_{\theta}=-g \sin \theta
\end{aligned}
$$

Relationship between kinematic viscosity and dynamic viscosity: 


$$
v=\frac{\mu}{\rho}
$$

Where, $v$ is the kinematic viscosity, $\mu$ is the dynamic viscosity, $\rho$ is the density.

Substituted formula (8), (11), (12), (13), (14), (15), (16) into equation (9) and (10):

$$
\left\{\begin{array}{l}
g \cos \theta=\frac{u_{\theta}^{2}}{r} \\
\rho g \sin \theta=\mu\left(\frac{\partial^{2} u_{\theta}}{\partial r^{2}}+\frac{1}{r} \frac{\partial u_{\theta}}{\partial r}-\frac{u_{\theta}}{r^{2}}\right)
\end{array}\right.
$$

Relationship between stress and strain change rate:

$$
\begin{aligned}
\frac{\partial \tau_{r \theta}}{\partial r}+2 \frac{\tau_{r \theta}}{r} & =\mu\left(\frac{\partial^{2} u_{\theta}}{\partial r^{2}}-\frac{1}{r} \frac{\partial u_{\theta}}{\partial r}+\frac{u_{\theta}}{r^{2}}+\frac{2}{r} \frac{\partial u_{\theta}}{\partial r}-\frac{2 u_{\theta}}{r^{2}}\right) \\
& =\mu\left(\frac{\partial^{2} u_{\theta}}{\partial r^{2}}+\frac{1}{r} \frac{\partial u_{\theta}}{\partial r}-\frac{u_{\theta}}{r^{2}}\right)
\end{aligned}
$$

Partial derivative of $\tau_{r \theta}$ :

$$
\frac{\partial \tau_{r \theta}}{\partial r}=\mu \frac{\partial}{\partial r}\left(\frac{\partial u_{\theta}}{\partial r}-\frac{u_{\theta}}{r}\right)=\mu\left(\frac{\partial^{2} u_{\theta}}{\partial r^{2}}-\frac{1}{r} \frac{\partial u_{\theta}}{\partial r}+\frac{u_{\theta}}{r^{2}}\right)
$$

And,

$$
\frac{\tau_{r \theta}}{r}=\mu\left(\frac{1}{r} \frac{\partial u_{\theta}}{\partial r}-\frac{u_{\theta}}{r^{2}}\right)
$$

According to formula (19) and (20):

$$
\begin{aligned}
\frac{\partial \tau_{r \theta}}{\partial r}+2 \frac{\tau_{r \theta}}{r} & =\mu\left(\frac{\partial^{2} u_{\theta}}{\partial r^{2}}-\frac{1}{r} \frac{\partial u_{\theta}}{\partial r}+\frac{u_{\theta}}{r^{2}}+\frac{2}{r} \frac{\partial u_{\theta}}{\partial r}-\frac{2 u_{\theta}}{r^{2}}\right) \\
& =\mu\left(\frac{\partial^{2} u_{\theta}}{\partial r^{2}}+\frac{1}{r} \frac{\partial u_{\theta}}{\partial r}-\frac{u_{\theta}}{r^{2}}\right)
\end{aligned}
$$

Substituted formula (21) into equation (17):

$$
\left\{\begin{array}{l}
\frac{u_{\theta}^{2}}{r}=g \cos \theta \\
\frac{\partial \tau_{r \theta}}{\partial r}+2 \frac{\tau_{r \theta}}{r}=\rho g \sin \theta
\end{array}\right.
$$

Formula (22) is the dynamics differential equations of magneto-rheological fluids in the MR dynamometer.

As we can see, the flowing state of magneto-rheological fluids and the working status of MR dynamometer can be reflected by the dynamics differential equations and the torque formula. Also they can show how $u_{\theta}$ and $T_{r \theta}$ will change with changing material properties or the dimensions of MR dynamometer.

\section{Conclusion}

1) MR dynamometer introduced magneto-rheological fluid as power transmission medium, making it simple structure, responsive, easy to control. It is a kind of new type dynamometer that having a broad prospect.

2) The torque magneto-rheological dynamometer absorbed is controlled by the current of excitation coil. The magnetic field strength of work space changes by adjusting the excitation current, then the magneto-rheological fluid generates MR effect, adjusting the shear stress, thereby achieving the purpose of adjusting the transmission torque.

3) This study analyses the principle of magneto-rheological dynamometer based on Bingham viscoplastic flow model, designed and constructed the magneto-rheological dynamometer test bench. The magneto-rheological dynamometer dynamic model is also established by using Magneto-rheological fluid constitutive model and fluid momentum equation, reflecting the torque transmission and the working condition of magneto-rheological fluids, and the effects of dynamometer structure and physical properties of MRF on the dynamometer torque transmission.

\section{Acknowledgements}

This research was supported by the construction of professional platform for modern automobile service engineering in Shanghai city (XKCZ1214).

\section{References}

[1] Jolly M R, Bender J W, Carlson D J. "Properties and applications of commercial magneto rheological fluids", Journal of Intelligent Material Systems and Structures, 2000, 10(11): 5-13.

[2] Wang Jianxiao, Meng Guang. "Magneto-rheological fluid devices and their applications in mechanical engineering", Journal of Mechanical Strength, 2001, 23(1): 50-56.

[3] Yi Chengjian. "Magneto-rheological Fluids: Preparation, Property and Modeling", Chongqing University, Chongqing, China, 2011.

[4] Zheng Jun, Zhang Guanghui, Cao Xingjin. "Flow analysis of magneto-rheological fluid in transmission device in steady state", Journal of Central South University (Science and Technology), 2008, 39(1): 149-154.

[5] Kamath G M, Hurt M K, Wereley N M. "Analysis and testing of Bingham plastic behavior in semi-active electro-rheological fluid dampers", Smart Material Structure, 1996, 10(5): 576-590.

[6] Li Hongnan, Yang Hao, Li Xiuling. "Advances of research on parameterized dynamic models of magneto-rheological dampers", Journal of Dalian University of Technology, 2004, 44(4): 616-624.

[7] LI Zhanwei, LI Zhijun. "Status of researching on dynamical models of MR damper", Machine Building \& Automation, 2012, 41(1): 142-145. 
[8] Liu Zhongliang, "Research on establishing new MRD dynamic model and numerical analysis", Central South University, Changsha, China, 2012.

[9] Meng Weijia, "The study and design of double-plates MRF clutch”, Harbin Institute Of Technology, Harbin, China, 2006.

[10] $\mathrm{Hu}$ Hongsheng, Wang Jiong, Qian Suxiang, Li Yancheng, Shen Na, Yan Gongbiao. "Dynamic Modeling and Its Sliding Controller of MR Shock Absorber under Impact Load", Journal of Mechanical Engineering, 2011, 47(13): 84-91.

[11] Liu Cheng, He Jianmin, Huangjin. "Analysis of Cylindrical Magneto-rheological Brake", Journal of Chongqing Institute of Technology (Natural Science), 2009, 23(7): 69-72.

[12] He Jianmin, Hung Jin, Zhong Yinhui. "Viscoplastic flow of the MR fluid between two cylinders", Function Materials, 2006, 37(6): 992-993.

\section{Biography}

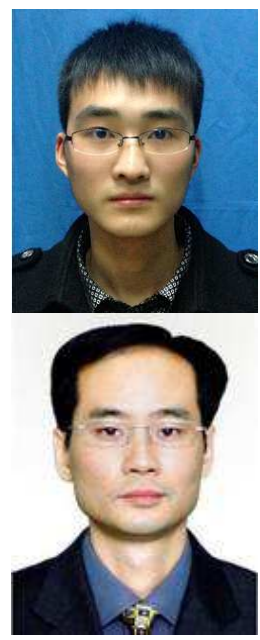

Tian Pengfei, Bachelor degree in engineering, Studying for Master Degree in Shanghai University of Engineering Science. The author's major is Vehicle Engineering.

Luo Yiping, University professor of Shanghai University of Engineering Science, Senior Engineer. The author's major is Automobile Manufacturing and Testing
[13] Tian Chaoyang, Zhang Jiangtao, Guo Zhijun, Wang Zhiwei. "Theoretical Design and Simulation Analysis of Disc MRF Brake”, Tractor \& Farm Transporter, 2014, 41(3): 19-21.

[14] Usob L E E, Doh Y K, Nahmkeon H. "Design analysis and experimental evaluation of an MR fluid clutch", Journal of Intelligent Material Systems and Structures, 1999, 10(9): 701-707.

[15] Ginder J M, Davis L C. "Shear stresses in magneto-rheological fluids: Role of magnetic saturation”, Appl Phys Lett, 1994, 65(26): 3410-3418.

[16] Weng Jiansheng, Hu Haiyan, Zhang Miaokang. "Experimental and modeling study on rheological properties of a kind of magneto-rheological fluid", Chinese Journal of Applied Mechanics, 2000, 17(3): 1-5. 